\title{
Aspectos a considerar en los cálculos de efectividad de una cobertura de valor razonable en donde el swap de tasa de interés intercambia una tasa flotante por otra tasa flotante (Key points to consider during the implementation of the effectiveness testing for a variable-to-variable interest rate swap hedging relationship)
}

\author{
Miguel A. García, Heriberto G. Martínez \& Jesús G. Cruz \\ UANL, San Nicolás, N.L., México, mgmrdg@yahoo.com.mx
}

Key words: Bulletin C-10, effectiveness testing, hedge accounting, swaps

\begin{abstract}
This article analyzes the hedge accounting of fair value hedge on a variable-tovariable swap. Following the effectiveness valuation procedure established in Bulletin C-10 "Derivative Financial Instruments and Hedge Activities" under Mexican GAAP will generate distorted effectiveness outcome even though the critical terms between the hedge item and the hedging instrument are the same in all aspects. To show this effect we present a hypothetic example for a fair value hedge relationship LIBOR-TIIE. Our conclusion is that this distortion is due to a valuation effect known as "pull to par" especially when the coupon reset dates of the hedge item and the hedging instrument does not match with the valuation date. Finally, it propones alternative ways to book this kind of transactions that eliminate the temporal distortion that this pull to par effect generates.
\end{abstract}

Palabras claves: Boletín C-10, contabilidad de coberturas, evaluación de efectividad, swaps

Resumen. En este artículo se analiza que la contabilización de una cobertura de valor razonable de un swap de tasa de interés con intercambios de variable por variable, siguiendo el procedimiento de evaluación de efectividad establecido en el Boletín C-10 "Instrumentos Financieros Derivados y Operaciones de Cobertura" de las Normas de Información Financieras, generará resultados de efectividad distorsionados aún y cuando los términos críticos entre la Posición Primaria sujeta a cobertura y el Instrumento Financiero Derivado (IFD) utilizado sean iguales. Para demostrar este efecto se desarrolla un ejemplo hipotético de una cobertura de valor razonable en donde se intercambian tasas LIBOR por TIIE. Se comenta que esta distorsión se genera debido a un efecto conocido como "pull to par" especialmente cuando la fecha de valuación no coincide con la fecha de corte de cupón del 
Instrumento Financiero Derivado y de la Posición Primaria. Finalmente se propone la contabilización para este tipo de coberturas contables que permita eliminar la distorsión temporal que se genera.

\section{Introducción}

Durante la última década la operación con Instrumentos Financieros Derivados (IFD) en México se han venido sofisticando día a día. En un inicio la operación de estos instrumentos, en la mayoría de las ocasiones, era exclusiva entre Instituciones Financieras, sin embargo, las entidades no financieras han descubierto los enormes beneficios que tienen estos instrumentos si son utilizados correctamente.

Es evidente que durante muchos años las reglas de contabilización para este tipo de instrumentos no eran las adecuadas debido a que los IFD no se reconocían en el balance general o solamente se reconocían los valores que se estaban devengando como efecto de valuación. Los IFD han experimentado en la última década una metamorfosis en todos sus aspectos, al pasar de IFD "Plain Vanilla" a IFD extremadamente complejos y volátiles. La velocidad con la que las áreas de negocio de las instituciones financieras desarrollan nuevos productos es por mucho más rápida, a la velocidad con la que los organismos encargados de promulgar los criterios contables establecen nuevas reglas. Uno de los problemas más evidentes era no contar con reglas claras de contabilización que permitiera de alguna manera cuantificar el efecto de valuación a futuro de estos instrumentos. Con la finalidad de establecer los primeros pasos para cerrar esta brecha, fue necesario crear un marco conceptual en donde se definieran reglas de reconocimiento, valuación, clasificación, revelación y medición para este tipo de IFD.

En el prologo de la octava edición de los principios de contabilidad generalmente aceptados se menciona "Las normas contables son dinámicas. Se van adaptando a los cambios experimentados en el entorno bajo el cual desarrollan su actividad las empresas. Esto es, algunas reglas se modifican, otras se eliminan o bien simple y sencillamente, surge la necesidad de emitir nuevas disposiciones. Esto hace necesario que con cierta periodicidad se publiquen ediciones nuevas debidamente actualizadas..." (IMCP, 1992).

M.A. García et al. 


\section{Antecedentes Normativos}

Hasta diciembre del 2000, el Boletín que de alguna manera definía el marco conceptual para los IFD era el Boletín C-1 "Efectivo e Inversiones temporales". La definición de inversiones temporales era "Las inversiones temporales están representadas por valores negociables o por cualquier otro tipo de inversión, convertibles en efectivo en el corto plazo y tienen por objeto, normalmente, obtener un rendimiento hasta el momento en que estos recursos sean utilizados por la entidad. Los valores negociables son aquellos que se cotizan en bolsas de valores o son operados a través del sistema financiero" (IMCP, 1992)

Las reglas de valuación establecidas en aquel entonces requerían que las inversiones en valores negociables se registraran a su valor neto de realización, el cual era representado por el valor de cotización en el mercado 0 el precio de venta de la inversión, menos los gastos que se hubiesen incurrido en dicha venta. Este Boletín C-1 que tuvo vigencia a partir del $1^{\circ}$ de enero de 1990 dejo sin efecto a las disposiciones contenidas en los Boletines C-1 "Efectivo" y C-2 "Inversiones Temporales" que se venían aplicando hasta ese entonces. Bajo las disposiciones del boletín de inversiones temporales únicamente se consideraban como inversiones temporales en valores negociables, aquellos valores que se cotizaban y operaban regularmente en bolsa de valores.

En diciembre de 1999 la Comisión de Principios de Contabilidad (CPC) emite el Boletín C-2 "Instrumentos Financieros" (C-2), aplicable a partir del $1^{\circ}$ de enero de 2001, con la finalidad de establecer un marco de referencia que permita dar soluciones a las siguientes problemáticas que se venían presentando: (1) utilizar los valores de mercado de los activos y pasivos que son instrumentos financieros, (2) determinar el reconocimiento de las ganancias (pérdidas) generadas por las valuaciones, (3) establecer el momento en que una entidad debía reconocer los activos y pasivos derivados de un contrato o convenio que califica como instrumento financiero y (4) señalar como debían registrarse las coberturas de riesgos.

Es en este boletín en donde se estableció de manera formal las primeras referencias hacia los IFD. La definición que se tenía en aquel entonces de un IFD era: "...contratos que se celebran con el objeto de crear derechos y obligaciones a las partes que intervienen en los mismos y cuyo único propósito

\section{Contabilidad de Coberturas}


es transferir entre dichas partes uno o más de los riesgos - es la posibilidad de que ocurra algún evento 0 acontecimiento en el futuro que cambie las circunstancias actuales 0 esperadas, que han servido de fundamento a la valuación de activos financieros y pasivos financieros, y que de ocurrir dicho evento 0 acontecimiento puede ocasionar un pérdida o ganancia, o cambios en los flujos de efectivo futuros - asociados con un bien o con un valor subyacente (base de referencia para valuar el riesgo). Los instrumentos derivados no crean la obligación de transferir la propiedad del bien o valor subyacente al inicio del contrato y dicha transferencia no necesariamente se da al término del contrato". (NIF, 2004)

Bajo las disposiciones del C-2 los IFD debían ser valuados en el balance y reconocidos en el estado de resultados utilizando el mismo criterio de valuación y reconocimiento aplicado a los activos o pasivos cubiertos.

Sin embargo, estas disposiciones establecidas en el C-2 se vieron pronto rebasadas por la mayor complejidad que presentan los IFD cotizados en el mercado, lo que generó la necesidad de hacer un boletín contable más robusto que permitiera establecer reglas claras sobre la contabilización y clasificación de este tipo de instrumentos con la finalidad de reflejar una mejor información financiera a todos los interesados. Es por tal motivo que en enero de 2005 entra en vigor el Boletín C-10 "Instrumentos Financieros Derivados y Operaciones de Cobertura" (C-10) que tiene como objetivo lo siguiente (NIF, 2004, p.7):

- "Establecer las características que debe tener un instrumento financiero para ser considerado como derivado;

- Definir y clasificar los modelos de contabilización de operaciones con fines de cobertura;

- Establecer las condiciones que debe cumplir un instrumento financiero derivado para considerarse como instrumento de cobertura, así como señalar las condiciones que deben cumplir las posiciones primarias sujetas a ser designadas en una relación de cobertura;

- Definir el concepto de efectividad de cobertura y establecer las reglas relativas a su evaluación inicial y medición posterior, ya que se trata de la característica esencial que debe tener el instrumento financiero derivado para ser designado y documentado como instrumento de cobertura;

M.A. García et al. 
- Establecer las reglas de reconocimiento y valuación, presentación y revelación contenidas en el Boletín C-2, aplicables a los instrumentos financieros derivados, incluyendo aquéllos con fines de cobertura, y

- Establecer las reglas de reconocimiento y valuación, presentación y revelación aplicables a las operaciones de cobertura estructuradas a través de derivados".

Dentro de los múltiples objetivos que señala el C-10, el inciso d) hace mención al concepto de efectividad de cobertura, el cual es abordado en el cuerpo del boletín bajo dos términos: (1) evaluación de la efectividad, la cual puede ser prospectiva o retrospectiva y (2) medición de efectividad.

Uno de los cambios más importantes incorporados dentro del C-10 es el correspondiente a la definición de un IFD. Con esta nueva definición un instrumento financiero podrá ser considerado como derivado si reúne todas las siguientes características:

- "Tiene uno o más subyacentes e incorpora uno o más montos nocionales o condiciones de pago, o ambos; dichos conceptos determinan el monto de la liquidación o liquidaciones y, en algunos casos, si se requiere o no liquidación;

- Requiere una inversión neta inicial nula o pequeña respecto a otro tipo de contratos que incorporan una respuesta similar ante cambios en las condiciones de mercado; $y$

- Sus términos requieren o permiten una liquidación neta. El contrato puede ser liquidado de manera neta a través de medios o mecanismos que se encuentran fuera del mismo, o bien, estipula la entrega de un activo que coloca al receptor en una posición sustancialmente similar a la liquidación neta".

Como se puede apreciar, con esta nueva definición de IFD lo que se buscó fue generar un marco de referencia más amplio, con la finalidad de que un instrumento financiero sea clasificado como derivado por sus propias características y ya no por su nombre como se venía considerando anteriormente.

EI C-10 establece que los IFD pueden ser clasificados como: (a) fines de negociación o (b) fines de cobertura. Para esta última opción se establecen los requisitos de documentación para que un IFD pueda ser designado bajo alguna de las alternativas de cobertura contable establecidas por la norma, las cuales son: (1) de valor razonable; (2) de flujo de efectivo y (3) de moneda extranjera.

\section{Contabilidad de Coberturas}


Independientemente de la clasificación contable que se le dé al IFD, todos los IFD deben ser valuados a su valor razonable, entendiéndose como valor razonable "la cantidad por la cual puede intercambiarse un activo financiero, 0 liquidarse un pasivo financiero, entre partes interesadas y dispuestas, en una transacción en libre competencia." (NIF, 2004)

Los requisitos de documentación (designación formal) que se deben cumplir para clasificar un IFD como de cobertura son los siguientes:

- La estrategia, el objetivo y su justificación para llevar a cabo la operación desde una perspectiva de administración del riesgo.

- La identificación de la posición primaria sujeta a cobertura y el riesgo o los riesgos que se pretenden cubrir de ella.

- Una descripción de las características del IFD o no derivado que será utilizado con fines de cobertura.

- El método que la entidad utilizará para llevar a cabo las pruebas de evaluación (prospectiva y retrospectiva) y de medición de efectividad. Estas pruebas deben ser realizadas al inicio y durante la vida de la cobertura con una periodicidad por lo menos trimestral.

- El tratamiento contable que se llevará a cabo en caso de excluir algún componente de la valuación al momento de realizar las pruebas de efectividad.

Uno de los requisitos de la evaluación de la efectividad, es que la relación de cobertura debe ser altamente efectiva, entendiéndose por altamente efectiva en donde los cambios en el valor razonable o en los flujos de efectivo de la posición primaria, son compensados por los cambios en el valor razonable o en los flujos de efectivo del instrumento de cobertura en una razón que fluctúe entre el $80 \%$ y $125 \%$.

Por otro lado el C-10, dentro de su párrafo 67 (NIF, 2004, p.27) hace mención a lo siguiente "Si las características críticas del instrumento de cobertura y de la posición primaria son iguales (el monto nocional, tasas de referencia para pago y cobro, y las bases relacionadas, la vigencia del contrato, la fecha de fijación de precio y de pago, las fechas de concertación y liquidación, entre otras), entonces los cambios en el valor razonable o en los flujos de efectivo atribuibles al riesgo que se está cubriendo, se compensarán completamente al inicio, durante y hasta el vencimiento de la cobertura, por lo cual no será necesario evaluar y medir la efectividad".

M.A. García et al. 
Si los IFD no cumplen con los "términos críticos" comentados en el párrafo anterior, entonces es necesario que la compañía realice de manera trimestral las pruebas de evaluación de efectividad, las cuales, como comentamos anteriormente, son: (1) prospectivas y (2) retrospectivas, así como su correspondiente medición de efectividad para aquellas coberturas que hayan sido clasificadas como de flujo de efectivo.

En el estudio realizado por (Bodurtha, 2005) se analiza si matemáticamente es válido asumir que un espejo de las características de la posición primaria con el IFD no generará inefectividad, debido a que los términos cualitativos son iguales, término conocido en USGAAP como "Short Cut Method". Este estudio expone una de las principales diferencias que existen entre la normatividad contable americana (FASB, F. A. 2004) y la normatividad contable internacional (IFRS, I. A. 2005). En opinión de Bodurtha la mejor forma de remediar el problema de inefectividad que se presenta aún y cuando los términos críticos sean los mismos, es utilizando el término del "derivado hipotético" y realizando pruebas de efectividad. Otras investigaciones (Coughlan, Kolb, \& Emery, 2003), mencionan que una manera de resolver este problema temporal de inefectividad es utilizando precios limpios, es decir, eliminando los intereses devengados del cupón vigente y realizando la prueba de efectividad .

Uno de los mayores problemas a los que se encuentran sujetos los preparadores de la información financiera es que el C-10 no contiene una explicación detallada sobre los conceptos y términos utilizados a lo largo del cuerpo del boletín. Esto ha originado que la aplicación de los conceptos haya sido incorrecto, incompletos y en algunas situaciones no se hayan aplicado, motivo por el cual el Centro Mexicano para la Investigación y Desarrollo de Normas de Información Financiera, A.C. (CINIF) ha promulgado algunas Interpretaciones a las Normas de Información Financiera (INIF) sobre el C-10, así como, Orientaciones a las Normas de Información Financiera (ONIF) con la finalidad de aclarar la interpretación y aplicación del C-10.

Es evidente que la complejidad de los IFD que se utilizan hoy en día ha requerido del establecimiento de una norma contable compleja, en donde se establezcan una serie de requisitos indispensables con la finalidad de poder establecer un marco normativo robusto sobre la forma de contabilizar dichos instrumentos. 


\section{Problema}

El C-10 menciona que una cobertura de variable a variable califica bajo una contabilización de cobertura de valor razonable, debido a que no se han mitigado todos los riesgos a los que se encuentra expuesta la entidad. Bajo este método de contabilización la posición primaria sujeta a cobertura deberá ser valuada a valor razonable, sin embargo, dicho procedimiento generará que las pruebas de efectividad arrojen resultados por afuera del límite establecido de efectividad del $80 \%$ al $125 \%$ en aquellas fechas en las que el corte de cupón no corresponde con la fecha de valuación.

\section{Alcance}

Derivado de lo anterior, el presente trabajo de investigación tiene como propósito general de estudio demostrar mediante la elaboración de un ejercicio hipotético que efectivamente las pruebas de efectividad rompen el límite establecido de 80/125 cuando el corte de cupón no corresponde con la fecha de valuación y por lo tanto proponer una solución que permita eliminar la distorsión que esta diferencia en fechas ocasiona temporalmente.

\section{Materiales y métodos}

Para entender la esencia del problema que se tiene en una cobertura de variable a variable, es importante demostrar a la fecha de inicio y subsecuentes fechas de corte de cupón el valor razonable del derivado siempre será igual a su valor nominal, efecto conocido como "pull-to-par" (Tuckman \& Jegadeesh, 2000).

A continuación, se presenta la comprobación matemática que al inicio y en fechas subsecuentes de corte de cupón el valor razonable del instrumento financiero que paga cupones durante la vida del instrumento será igual a su valor nominal (véase la ecuación No. 1).

M.A. García et al. 


$$
V F=V N * \frac{\left[1+T_{c} * \frac{P_{c}}{360}\right] *\left[1+\left(\frac{1+T_{l} * \frac{P_{l}}{360}}{1+T_{c} * \frac{P_{c}}{360}}-1 * \frac{360}{P_{l}-P_{c}}\right)\right] * \frac{P_{c}}{360}}{1+T_{l} * \frac{P_{l}}{360}}
$$

Donde:

VF: $\quad$ Valor futuro

VN: Valor nominal

$\mathrm{T}_{\mathrm{c}}$ : $\quad$ Tasa nominal de mercado al plazo de inicio

$P_{c}$ : $\quad$ Plazo en días al inicio del plazo acordado

$\mathrm{T}_{\mathrm{l}}$ : $\quad$ Tasa nominal de mercado al plazo de vencimiento

$\mathrm{P}_{\mathrm{l}}$ : $\quad$ Plazo en días al final del plazo acordado

Después de realizar una simplificación algébrica podemos concluir que en la fecha de corte de cupón, el Valor Futuro es igual al Valor Nominal, es decir $V F=V N$.

Para demostrar lo antes mencionado, se presenta el siguiente caso de una cobertura de valor razonable. Compañía $X$ contrata un Cross Currency Swap (CCS) mediante el cual paga tasa de interés variable (TIIE) y recibe variable (LIBOR) con la finalidad de cubrir una emisión de deuda en moneda extranjera a tasa variable (LIBOR). Las características del instrumento financiero derivado y la posición primaria son los siguientes:

\begin{tabular}{|l|l|l|}
\hline Concepto & Derivado & Posición Primaria \\
\hline Nocional MXN & $\$ 100,000,000$ & N/A \\
\hline Nocional USD & $\$ 9,267,239$ & $\$ 9,267,239$ \\
\hline Tasa Variable Recibo & LIBOR & N/A \\
\hline Tasa Variable Pago & TIIE & LIBOR \\
\hline Fecha de Inicio & Septiembre 30, 2005 & Septiembre 30, 2005 \\
\hline Pago de Cupón & Cada 180 días & Cada 180 días \\
\hline Fecha de Vencimiento & Septiembre 30, 2010 & Septiembre 30, 2010 \\
\hline
\end{tabular}

En el ejemplo anterior, los términos críticos del derivado y de la posición primaria son iguales y por lo tanto aplicando el párrafo 67 del C-10 se

\section{Contabilidad de Coberturas}


podría concluir que no existe inefectividad en la relación de cobertura, sin embargo, como se demostrara en la siguiente sección, los cálculos de las pruebas de efectividad arrojan resultados diferentes.

\section{Resultados}

Para llevar a cabo las pruebas de valuación del IFD y de la Posición Primaria antes descritos, se realizo lo siguiente:

1. Se realizaron valuaciones del IFD y de la Posición Primaria a precio sucio, es decir incluyendo los intereses devengados del cupón, y a precio limpio, en las siguientes fechas: 30/09/05, 31/12/05, 31/12/06 y $31 / 12 / 07$.

2. Se realizaron valuaciones del IFD y de la Posición Primaria en las siguientes fechas de corte de cupón: 30/09/05, 30/03/06 y 30/09/06

3. Como insumos se utilizó la curva LIBOR simple y la curva Descuento IRS publicada por Proveedor de Precios autorizado por la Comisión Nacional Bancaria y de Valores (CNBV) para las fechas antes mencionadas. Para el tipo de cambio MXN/USD se utilizó el FIX determinado por Banco de México.

4. Se utilizo el modelo de valuación para Swaps de divisa de tasas de interés variables o fijas utilizado por la industria financiera para determinar el valor razonable de este tipo de IFD, mismo que se muestra a continuación:

\section{Swaps de divisa de tasas de interés variables o fijas}

Son contratos mediante los cuales se establece la obligación bilateral de intercambiar una serie de pagos en efectivo por un periodo determinado, sobre un principal nocional denominado en moneda nacional y extranjera, ya que expresa los flujos de efectivo por diferenciales en las tasas de interés fija y variable tanto en moneda nacional como en divisas según se muestra en la siguiente ecuación (véase la ecuación No. 2):

Ecuación No. 2.
$M T M=B d\left[\sum_{i=1}^{n} \frac{\left(r_{d} * p / 360\right)}{\left(1+r_{d i} * t_{m i} / 360\right.}+\frac{1}{\left(1+r_{d n} * t_{m n} / 360\right)}\right]-B_{f} * S *\left[\sum_{i=1}^{n} \frac{\left(r_{f} * p / 360\right)}{\left(1+r_{f i} * t_{m i} / 360\right)}+\frac{1}{\left(1+r_{f n} * t_{m n} / 360\right)}\right]$

M.A. García et al. 
En el caso de recibir tasa pasiva extranjera, el cálculo del valor a mercado se realiza con base en la siguiente ecuación (véase la ecuación No. 3):

$$
\begin{aligned}
& \text { Ecuación No. 3. } \\
& M T M=B_{f} * S *\left[\sum_{i=1}^{n} \frac{\left(r_{f} * p / 360\right)}{\left(1+r_{f i} * t_{m i} / 360\right)}+\frac{1}{\left(1+r_{f n} * t_{m n} / 360\right)}\right]-B d\left[\sum_{i=1}^{n} \frac{\left(r_{d} * p / 360\right)}{\left(1+r_{d i} * t_{m i} / 360\right)}+\frac{1}{\left(1+r_{d n} * t_{m n} / 360\right)}\right]
\end{aligned}
$$

Donde:

MTM: Valor a mercado de la posición

Bd: Deuda en moneda nacional

Bf: Deuda en moneda extranjera

$r_{d}$ : Tasa doméstica

$r_{\mathrm{f}} \quad$ Tasa foránea

$\mathrm{n}$ : $\quad$ Número de períodos vigentes en que se realizarán pagos de interés

$t_{\mathrm{mi}}$ : $\quad$ Días por vencer del período $\mathrm{i}$

$r_{\mathrm{di}}: \quad$ Tasa en moneda nacional al plazo de vencimiento $t_{\mathrm{mi}}$

$\mathrm{r}_{\mathrm{fi}}: \quad$ Tasa en la divisa pactada al plazo de vencimiento $t_{\mathrm{mi}}$

$\mathrm{p}$ : $\quad$ Plazo de referencia del swap de divisas

S: $\quad$ Tipo de cambio spot vigente al momento de la valuación

A continuación se presentan los resultados obtenidos del ejercicio hipotético realizado utilizando precios sucios (véase la tabla No. 1).

\begin{tabular}{|c|c|c|c|c|c|c|}
\hline $\begin{array}{c}\text { Fecha de } \\
\text { Valuación }\end{array}$ & $\begin{array}{c}\text { Valor } \\
\text { Razonable } \\
\text { Derivado }\end{array}$ & Cambio & $\begin{array}{c}\text { Tabla 1. } \\
\text { Razonable } \\
\text { Posición } \\
\text { Primaria }\end{array}$ & Cambio & $\begin{array}{c}\text { Razón de } \\
\text { Cobertura }\end{array}$ & $\begin{array}{c}\text { Razón } \\
\text { Acumulada }\end{array}$ \\
\hline $\begin{array}{c}\text { Septiembre } \\
2005\end{array}$ & 0 & & $-100,000,000$ & & & \\
\hline $\begin{array}{c}\text { Diciembre } \\
2005\end{array}$ & $-3,014,940$ & $-3,014,940$ & $-99,532,822$ & 467,178 & $-645 \%$ & $645 \%$ \\
\hline $\begin{array}{c}\text { Diciembre } \\
2006\end{array}$ & $-1,808,471$ & $1,206,469$ & $-100,987,590$ & $-1,454,767$ & $-83 \%$ & $-183 \%$ \\
\hline $\begin{array}{c}\text { Diciembre } \\
2007\end{array}$ & 605,504 & $2,413,974$ & $-102,489,295$ & $-1,501,705$ & $-161 \%$ & $-24 \%$ \\
\hline
\end{tabular}


Como se puede apreciar la razón de cobertura se encuentra, tanto de manera periódica como acumulada, fuera del rango de tolerancia establecido de $80 / 125$, al presentarse resultados de $645 \%, 83 \%$ y $161 \%$ al cierre de diciembre de 2005, 2006 y 2007, respectivamente. Dichos resultados se obtienen al comparar el cambio en el valor razonable del IFD versus el cambio en el valor razonable de la Posición Primaria.

Asimismo, si se realizan las mismas valuaciones a precios limpios, los resultados obtenidos mediante la realización del ejercicio hipotético presentan una razón de cobertura más cercana al rango establecido del $80 / 125$, como se muestra en la siguiente tabla (véase la tabla No. 2).

\begin{tabular}{|c|c|c|c|c|c|c|}
\hline $\begin{array}{l}\text { Fecha de } \\
\text { Valuación }\end{array}$ & $\begin{array}{c}\text { Valor } \\
\text { Razonable } \\
\text { Derivado }\end{array}$ & Cambio & $\begin{array}{c}\text { Valor } \\
\text { Razonable } \\
\text { Posición } \\
\text { Primaria }\end{array}$ & Cambio & $\begin{array}{l}\text { Razón de } \\
\text { Cobertura }\end{array}$ & $\begin{array}{c}\text { Razón } \\
\text { Acumulada }\end{array}$ \\
\hline $\begin{array}{c}\text { Septiembre } \\
2005\end{array}$ & - & & $-100,000,000$ & & & \\
\hline $\begin{array}{c}\text { Diciembre } \\
2005\end{array}$ & $-1,741,150$ & $-1,741,150$ & $-98,478,742$ & $1,521,258$ & $-114 \%$ & $114 \%$ \\
\hline $\begin{array}{c}\text { Diciembre } \\
2006\end{array}$ & $-544,451$ & $1,196,699$ & $-99,918,103$ & $-1,439,361$ & $-83 \%$ & $665 \%$ \\
\hline $\begin{array}{c}\text { Diciembre } \\
2007\end{array}$ & $1,126,218$ & $1,670,668$ & $-101,140,389$ & $-1,222,285$ & $-137 \%$ & $-99 \%$ \\
\hline
\end{tabular}

Si bien es cierto que los resultados de razón de cobertura mediante la utilización de precios limpios mejora considerablemente, dichos resultados arrojan también inconsistencias dado que para la evaluación periódica de diciembre de 2007 y acumulada de Diciembre de 2006, los resultados obtenidos se encuentra por afuera de los límites establecidos para que la relación de cobertura pueda ser considerada como altamente efectiva.

Sin embargo, si la prueba de efectividad se lleva a cabo en la misma fecha en la que el IFD y la Posición Primaria cortan cupón, los resultados obtenidos muestran una relación de efectividad del 100\% como se muestra en la siguiente tabla (véase la tabla No. 3).

Como se puede apreciar, a las fechas de corte de cupón la efectividad de la relación de cobertura es perfecta dado que los cambios en los valores razonables de la Posición Primaria y del IFD presentan los mismos valores.

M.A. García et al. 
Tabla No. 3.

\begin{tabular}{|c|c|c|c|c|c|c|}
\hline $\begin{array}{c}\text { Fecha de } \\
\text { Valuación }\end{array}$ & $\begin{array}{c}\text { Valor } \\
\text { Razonable } \\
\text { Derivado }\end{array}$ & Cambio & $\begin{array}{c}\text { Valor } \\
\text { Razonable } \\
\text { Posición } \\
\text { Primaria }\end{array}$ & Cambio & $\begin{array}{c}\text { Razón de } \\
\text { Cobertura }\end{array}$ & $\begin{array}{c}\text { Razón } \\
\text { Acumulada }\end{array}$ \\
\hline $\begin{array}{c}\text { Septiembre } \\
2005\end{array}$ & 0 & & $-100,000,000$ & & & \\
\hline $\begin{array}{c}\text { Marzo } \\
2006\end{array}$ & 952,672 & 952,672 & $-100,952,672$ & - & $-100 \%$ & $100 \%$ \\
\hline $\begin{array}{c}\text { Septiembre } \\
2006\end{array}$ & $1,879,396$ & 926,724 & $-101,879,396$ & - & $-100 \%$ & $-100 \%$ \\
\hline
\end{tabular}

\section{Conclusión:}

La diferencia temporal que se presenta en la medición de efectividad corresponde al efecto de la tasa del cupón corriente, por otro lado, hemos demostrado que para aquellas coberturas que mediante el uso de un derivado cambian una exposición de tasa variable a otra a tasa variable, en donde los términos críticos de la posición primaria y del derivado son idénticos, el valor futuro es igual al valor nominal por el efecto conocido como "pull-to-par", motivo por el cual, una cobertura de valor razonable que cambia la exposición de una posición primaria a tasa variable en moneda no funcional a una en tasa variable en moneda funcional, solamente puede ser sujeta a cobertura por el componente de tipo de cambio.

El procedimiento que establece el C-10 sobre cómo debe reconocerse una operación de cobertura de valor razonable en su párrafo 102 consideramos que es válido para aquellas coberturas que cambian una exposición de tasa fija hacia una de tasa variable, sin embargo, en nuestro ejemplo de variable a variable, el procedimiento al que se hace mención sobre obtener el valor razonable de la partida primaria no aplicaría, dado que ocasionaría "ruido" en la medición de la efectividad, por lo que solamente se tendría que estar reconociendo la ganancia (pérdida) por el efecto de fluctuación cambiaria.

Asimismo, si los términos críticos del derivado son idénticos en todos los aspectos con los de la posición primaria, el valor razonable del derivado, se tendría que ajustar con la finalidad de que su cambio compense al $100 \%$ la ganancia (pérdida) que se está calculando para la posición primaria en resultados. 
A continuación se muestra un ejemplo de cómo quedarían las entradas contables utilizando el procedimiento contable antes mencionado (véase la tabla No. 4):

\begin{tabular}{|c|c|c|c|c|c|}
\hline $\begin{array}{c}\text { Fecha de } \\
\text { Valuación }\end{array}$ & $\begin{array}{c}\text { MtM } \\
\text { Derivado }\end{array}$ & Cambio & \multicolumn{1}{c|}{$\begin{array}{c}\text { Posición } \\
\text { Primaria }\end{array}$} \\
$\begin{array}{c}\text { Septiembre } \\
2005\end{array}$ & - & & $-100,000,000$ & & \\
\hline $\begin{array}{c}\text { Diciembre } \\
2005\end{array}$ & $-1,741,150$ & $-1,741,150$ & $-98,551,530$ & $1,448,470$ & $-292,680$ \\
\hline
\end{tabular}

$---1--$

Cargo: Deuda Pasivo

$\$ 1,448,470$

Abono: Ganancia (pérdida) en cambios (P\&L)

$\$ 1,448,470$

(Para reconocer la ganancia por el efecto de fluctuación cambiaria al cierre de diciembre 2005)

$---2--$

Cargo: Ganancia (pérdida) en cambios (P\&L)

$\$ 1,741,150$

Abono: Operaciones con Derivados (Pasivo)

$\$ 1,741,150$

(Para reconocer la minusvalía del valor razonable del derivado al cierre de diciembre 2005)

$---3--$

Cargo: Operaciones con Derivados (Pasivo)

$\$ 292,680$

Abono: Ganancia o pérdida en cambios (P\&L)

$\$ 292,680$

(Para eliminar el efecto del "ruido" generado por el cupón corriente y compensar adecuadamente los efectos generados por la posición primaria sujeta a cobertura).

\section{Referencias}

Bodurtha, J. N., 2005. Divergent FAS-133 and IAS-39 Interest Rate Risk Hedge Effectiveness: Problem and Remedies. Journal of Derivatives Accounting , 2 (1), 1 13

Coughlan, G., Kolb, J., \& Emery, S., 2003. HEAT Technical Document: A consistent framework for assessing hedge effectiveness under IAS39 and FAS133. London: Credit \& Rates Markets J.P. Morgan Securities Ltd.

M.A. García et al. 
FASB, F.A., 2004. Accounting for Derivative Instruments and Hedging Activities. FASB Statement No. 133. Norwalk, Connecticut: Financial Accounting Standard Board.

Hull, J., 2008. Options, Futures \& Other Derivatives. USA: Prentice Hall.

IFRS, I.A., 2005. Financial Instruments, Reporting and Accounting. United Kingdom: International Accounting Standards Committee Foundation.

IMCP, I.M., 1992. Principios de contabilidad generalmente aceptados. México, D.F.: Instituto Mexicano de Contadores Públicos, A.C.

NIF, I.M., 2004. Principios de Contabilidad Generalmente Aceptados. México: Instituto Mexicano de Contadores Públicos, A.C.

Tuckman, B., \& Jegadeesh, N., 2000. Advanced Fixed- Income Valuation Tools. Canada: John Wiley \& Sons. 

